\title{
Inclusion Body Hepatitis and Hydropericardium Syndrome in Poultry: A Brief Review
}

\section{${ }^{1}$ Sumitra Panigrahi, ${ }^{2}$ Krutanjali Swain, ${ }^{1}$ Abhilash Routray, ${ }^{3}$ Adya Prakash Rath, ${ }^{4}$ Saraswat Sahoo and ${ }^{5}$ Subha Ganguly*}

${ }^{1}$ MVSc Scholar, Dept. of Veterinary Public Health and Epidemiology, LUVAS, ${ }^{2}$ MVSc Scholar, Dept. of Veterinary Parasitology, LUVAS, ${ }^{3}$ MVSc Scholar, Dept of Veterinary Pathology, LUVAS,

${ }^{4} \mathrm{Ph} . D$. Research Scholar, Dept of Animal Reproduction, Gynaecology \& Obstetrics, OUAT, Bhubaneswar, Odisha,

${ }^{5}$ Associate Professor, Dept of Veterinary Microbiology, Arawali Veterinary College (Affiliated with Rajasthan University of Vetrinary and Animal Sciences, Bikaner), Rajasthan, India

*Corresponding Author: Dr. Subha Ganguly,

\begin{abstract}
The inclusion body hepatitis (IBH) and the hydropericardium syndrome (HPS) of chickens have been etiologically associated fowl adenoviruses (FAVs) group 1, serotypes 1-12.The fowl adenoviruses (FAV112) are widely distributed in poultry flocks.The virus belongs to the genus Aviadenovirus under the family Adenoviridae. IBH and HPS are the most important disease conditions caused by this genus. Avian adenoviruses are classified in three groups (I to III).Different groups cause different diseases. These are eggdrop syndrome 76 in hens (group III), haemorrhagic enteritis of turkeys and marble spleen disease of pheasants (group II).All 12 serotypes have been incriminated in IBH, whereas serotype 4 has been implicated in HPS.The IBH/HPS affects chickens by inducing high mortality rates characterized by liver damage and hydropericardium. The hemopoietic system and the kidneys are also affected. The most important losses have been reported in 3-to-5-wk-old broilers; however, breeders and layers may also be affected.
\end{abstract}

Keywords: Aviadenovirus, Chicken, Egg drop syndrome, Inclusion body hepatitis,

\section{INTRODUCTION}

Inclusion body hepatitis (IBH) of chickens was first recognized in 1963. It normally occurs in meat-type chickens at 3 to 7 weeks of age, but it has been reported in birds as young as 7 dayold and as old as 20 weeks. High mortality occurs when the affected birds are less than three weeks of age. Depending on the pathogenicity of the virus, immune status of the chicks and concurrent secondary infections, mortality up to $80 \%$ has been reported (Howel et al., 1970; Monreal et al., 1980).

\section{HYDROPERICARDIAL SYNDROME}

In 1988 a new broiler disease was reported in Pakistan and called Angara Disease where there was sudden increase in mortality in healthy flocks at 3 to 5 weeks of age. Typical postmortem lesions included pulmonary edema, hepatitis and nephritis. The main pathological finding was the accumulation of a clear, straw colored fluid in the pericardial sac, therefore the disease was called hydropericardium syndrome (Cowen and Naki, 1982; Cheema et al., 1989).

\section{Transmission}

Horizontal and vertical transmission plays an important role in IBH/HPS.The virus transmits vertically through embryonated eggs.Horizontal spread occurs by contact with infected feces, 
fomites.Young chicks in contact with infected chicks can die of per acute IBH/HP (Rahimi and Haghighi, 2015).

\section{Pathogenesis}

After entering into body, initial multiplication of the virus occurs in both small and large intestine. Viraemia occurs with spreading of virus to many organs like liver, kidney, respiratory tract, bone marrow and bursa. Virus can be readily isolated from feces, ocular and nasal mucosa, bursa of fabricius. Chicken once affected with adenovirus becomes lifelong carrier. In layers, virus may be transmitted through egg (Anjum, 1990; Toro et al., 2000).

\section{Clinical Findings and Gross Lesions}

Sudden mortality is found in birds of 4 days old up to 6 weeks .Mortality generally ranges from 40-60\%. Typically, the mortality of HPS starts at three weeks of age and causes between $20-80 \%$ mortality. The main pathological findings of HPS are the accumulation of a clear, straw colored fluid in the pericardial sac. Classical IBH is characterized by focal hepatic necrosis and intranuclear inclusion bodies. Birds are found to be lethargic,hurdling with ruffled feather,yellow droppings.In almost all cases, lesions are found in liver and kidney. The kidneys are pale, swollen and mottled appearance. Hemorrhage into the renal cortex may be found in some of the birds (Cheema et al., 1989; Padhy et al., 2015).

\section{Histological Examination}

Livers show basophilic intranuclear inclusion bodies. Focal aggregation of round cell leucocytes is noted in some areas. Congestion and focal hemorrhage are observed in kidneys. In many of the hepatic cells, the nuclei disappear entirely, leaving a ghost cell consisting of one or more large vacuoles. Varying degrees of pyknosis, karyorrhexis and karyolysis are observed in the majority of the hepatic cells. Large Cowdry Type A intranuclear inclusion bodies are found in many of the degenerating liver cells. Myocardial edema in the heart with degeneration, necrosis, and mild mononuclear cell infiltration can be observed (Mazaheri et al., 1998; Padhy et al., 2015).

\section{DiAgnOSIS}

The laboratory diagnosis of adenovirus infections, including IBH in chickens in most cases based on histological investigations and detection of intra-nuclear inclusion bodies in hepatocytes or detection of the antigen or virus particles using immunofluorescence test or electron microscopy.

Virus detection can be confirmed by isolation of virus. The different serotypes can be determined by neutralization test (Erny et al., 1981).

\section{Prevention and Control}

The viruses like IBD and CIA viruses potentiate these diseases. So, the major step should be to eliminate these two agents. Appropriate biosecurity measures should be maintained.Infected and non-infected breeding flocks should be separated. Besides this, vaccination of the affected birds can be done. The AAV serotypes most frequently used to prepare commercial vaccines are serotypes 4 and 8 . Broilers are vaccinated at $<10$ days of age when their parents either do not have serotype-specific adenovirus antibodies (Cowen, 1992).

\section{REFERENCES}

[1] Anjum, A.D. (1990). Experimental transmission of hydropericardium syndrome and protection against it in commercial broiler chickens. Avian Pathology, 19: 655-660.

[2] Cheema, A.H., Ahmad, J. and Afzal, M. (1989). An adenovirus infection of poultry in Pakistan. Rev Sci Tech Off. Int Epiz. 8:789-795. 
[3] Cowen, B. (1992). Inclusion body hepatitis-anemia and hydropericardium syndromes: aetiology and control. Worlds Poult. Sci. J. 48:247-254.

[4] Cowen, B.S. and S.A. Naqi. (1982). Classification of avian adenoviruses. J. Am. Vet. Med. Assoc. 181: 283.

[5] Erny, K. M., Barr, D.A., and Fahey, K. J. (1991). Molecular characterization of highly virulent fowl adenoviruses associated with outbreaks of inclusion body hepatitis. Avian Pathology. 20:597-606.

[6] Howell, J., MacDonald, D.W. and Christian, R.G. (1970). Inclusion body hepatitis in chickens. The Canadian Veterinary Journal, 11:99.

[7] Mazaheri, A., Prusas, C., Voss, M. and Hess, M. (1998). Some strains of serotype 4 fowl adenoviruses cause inclusion body hepatitis and hydropericardium syndrome in chickens. Avian Pathology, 27:269-276.

[8] Monreal, G., Dorn, R. and Kassim, M. (1980). Bestimmung von Antikorperngegenaviare Adenovirenim Mikrotiter-Zellkultursystem. Berliner und Munchenertierarztliche Wochenschrift. 93:125-128.

[9] Padhy, Arpita, Sahu, Amit Ranjan, Sahoo, Saraswat and Ganguly, Subha (2015) Chicken infectious anemia: A Review on its potential threat to poultry industry worldwide. World J. Biol. Medi. Sci. 2(4): 17-19.

[10] Rahimi, M., and Haghighi, Z.M.S. (2015). Adenovirus-like inclusion body hepatitis in a flock of broiler chickens in Kermanshah province, Iran. Veterinary Research Forum.6: 95.

[11] Toro, H., Gonzalez, C., Cerda, L., Hess, M., Reyes, E. and Geisse, C. (2000).Chicken anemia virus and fowl adenoviruses: association to induce the inclusion body hepatitis/ hydropericardium syndrome. Avian diseases. 51-58. 